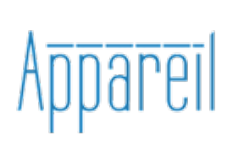

Appareil

$6 \mid 2010$

Philosophie et cinéma

\title{
Le Japon selon Chris Marker
}

Lieu du dépaysement temporel, entre le sommeil et le réveil

\section{Emi Koide}

\section{(2) OpenEdition \\ Journals}

Édition électronique

URL : http://journals.openedition.org/appareil/1107

DOI : 10.4000/appareil. 1107

ISSN : 2101-0714

Éditeur

MSH Paris Nord

Référence électronique

Emi Koide, « Le Japon selon Chris Marker », Appareil [En ligne], 6 | 2010, mis en ligne le 08 octobre 2010, consulté le 30 juillet 2020. URL : http://journals.openedition.org/appareil/1107 ; DOI : https:// doi.org/10.4000/appareil.1107

Ce document a été généré automatiquement le 30 juillet 2020.

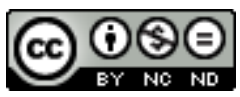

Appareil est mis à disposition selon les termes de la Licence Creative Commons Attribution - Pas d'Utilisation Commerciale - Pas de Modification 4.0 International. 


\section{Le Japon selon Chris Marker}

Lieu du dépaysement temporel, entre le sommeil et le réveil

\section{Emi Koide}

Après que la guerre est finie, nous sommes nés Nous sommes devenus adultes,

Nous commençons à marcher En chantant les chansons de paix Nous voulons que vous vous souvenez de notre

nom

Enfants qui ne connaissent pas la guerre Si on ne nous permet pas parce que nous sommes

trop jeunes

Si on ne nous permet pas parce qu'on a les cheveux longs

Ce qui me reste en ce moment est de chanter en

étant au bord des larmes

Nous voulons que vous vous souvenez de notre

nom

Enfants qui ne connaissent pas la guerre

(Extrait de paroles de la chanson « Sensô wo

shiranai kodomotachi »- Enfants qui ne connaissent pas la guerre - cité par Yoshikuni

Igarashi, $2001: 197)$

\section{Introduction}

1 Chris Marker, artiste multimédia, écrivain, photographe, "cinéaste", ou plutôt "bricoleur ", comme il préfère être appelé, a constamment exploré l'articulation des images, sons et textes en créant de nouvelles significations. La production markérienne a amené d'autres horizons à la production artistique de vidéo art et nouvelles technologies filmiques, en jouant entre la frontière de la fiction et de l'histoire, en les 
présentant en tension, pour questionner le cinéma, ses représentations, son rôle dans le monde. Contre les conventions et en échappant aux classifications, plusieurs de ses films se présentent comme une espèce de «travelogue » ou journaux de voyage, dans lesquels il présente, de façon critique, les idées de l'Occident sur l'Autre. De plus, il met en évidence les images clichées et stéréotypées, et par conséquent l'incapacité des films documentaires à faire le portrait d'une autre culture. Ses innombrables voyages nous renvoient à deux expériences initiatiques: la lecture de Jules Verne et la bandedessinée de la famille Fenouillard - selon lui les autres voyages avaient pour but de vérifier dans le propre lieu les enseignements des voyages littéraires (Guy Gauthier, $2001: 17)$

2 L'imaginaire et la subjectivité du voyageur entrent dans ses films, à l'opposé des documentaires d'histoire officielle, de sorte qu'il ne cherche pas à expliquer et réduire l'autre à celui qui est connu. À partir de ses voyages à travers le monde: Finlande, Chine, Sibérie, Corée, Israël, Cuba et Japon - pays où il revient plusieurs fois après - il a produit des films, photos et récits qui n'épuisent pas le sujet et qui ne donnent pas de discours linéaire. Sa fascination pour l'autre, se traduit dans son regard particulier vers l'Orient, surtout dans la figure du Japon. Dans la mesure où on identifie la récurrence de ce pays comme le lieu de l'imaginaire dans son œuvre. Selon Gauthier (2001:21), « la fascination pour le tour du monde a donc eu pour effet, entre autres, [... que] le Japon a sans cesse hanté son œuvre». Ou encore pour Möller (2003: 35), "Le Japon a une signification spéciale pour Marker », car il est revenu au pays de temps en temps en réalisant des œuvres. Sur cette île qui habite son imaginaire et sa mémoire, le narrateur markérien dit : "Inventer le Japon est un moyen comme un autre de le connaitre " (Marker, 1982).

3 Le présent texte propose de réfléchir sur le statut du Japon, en tant que territoire où se croisent la mémoire, l'imaginaire, le temps et l'espace - lieu de l'Altérité et du dépaysement. J'exposerai d'abord les différentes façons de voyager pour penser l'expérience du dépaysement. Ensuite, à partir de l'analyse des œuvres Le mystère Koumiko (1965), Sans Soleil (1982), Le Dépays (1982), Level Five (1997), j’étudierai comment cette expérience du dépaysement devient présente dans les films. Enfin, je proposerai l'idée selon laquelle les films donnent une vision du contexte du Japon de l'aprèsguerre, le phénomène d'une amnésie collective hanté par le fantôme de la guerre et son rapport avec le monde médiatique d'images.

\section{Différentes façons de voyager}

4 En considérant le rapport entre les différentes façons de voyager selon des conceptions d'espace et de temps, Sérgio Cardoso (1989) et Olgária Matos (1997) distinguent le voyage du géomètre où le déplacement a lieu dans un espace ordonné et homogène, marqué par l'isotropie, et le voyage du géographe qui se caractérise par l'établissement des frontières, en mesurant les distances. Mais, ces deux manières de voyager considèrent l'espace comme extension, c'est-à-dire selon une conception qui unifie tout, où il n'y a pas vraiment de distance car elle présuppose une continuité spatiale comme succession. En effet, cette idée d'espace est liée, à son tour, à une conception temporelle nécessairement linéaire, comme une succession des instants. Étant donné que le voyage est considéré comme un déplacement dans l'espace, définition qu'on trouve dans les dictionnaires, où il y a un mouvement, il s'agit d'un parcours selon un 
trajet défini où il faut un point de départ et d'arrivée, sans lesquels il n'est pas possible de le représenter. De sorte que cet espace-temps de succession ignore la distance, car elle est composée d'instants et points qui viennent les uns après les autres. Dans la mesure où il est nécessaire de connaitre et prédéterminer les points de trajet, il n'est pas possible d'envisager l'Altérité, ni pour les ouvertures, ni pour les indéterminations. Ainsi, cette conception du voyage comme simple déplacement dans l'espace est-elle réductrice :

[...] représenter naïvement ces mouvements comme déplacements entre lieux à l'intérieur d'un monde; ne permet pas de comprendre que le voyageur s'éloigne parce qu'il se différencie et transforme son monde; que les voyages sont toujours des entreprises dans le temps (Sérgio Cardoso, $1989: 358$ ).

Les vrais voyages pour Cardoso et Matos sont ceux où on peut trouver des ouvertures, dans lesquels il y a une expérience d'étrangeté, de dépaysement. Cependant, il ne s'agit pas d'apercevoir «l'étrangeté du monde entourant» et extérieur, mais de "signaler toujours des dérangements intérieurs dans le propre territoire du voyageur ", car c'est le temps qui transforme le voyageur qui « devient étrange à soi-même» (Ibid.: 359). C'est la temporalité qui entre dans le sujet voyageur pour qu'il puisse comprendre et faire l'expérience de l'altérité, où l'autre n'est pas un étranger complet qui appartient à l'extériorité mais qui se trouve dans notre intériorité même. Alors, il faut prendre de la distance et s'éloigner de soi-même pour voyager et avoir l'expérience du dépaysement. Selon Matos (1997 : 144-146), cette façon de voyager est celle du flâneur et de l'enfant benjaminien, où la ville devient une topographie de la mémoire pour se déplacer dans le temps, en établissant des rapports entre le passé et le présent, pas comme un trajet linéaire mais comme une constellation où " ne pas s'orienter dans une ville ne signifie pas grand chose, mais se perdre dans une ville, demande tout un apprentissage ». (Walter Benjamin, 1997 :73)

\section{Japon et le dépaysement temporel - entre le sommeil et le réveil}

6 Voyager en se dépaysant est aussi la façon pour Marker de faire le tour du monde, surtout dans les endroits et pays plus éloignés comme le Japon. Il nous apparaît ici nécessaire de revenir sur l'atmosphère qui caractérise le Japon de l'après-guerre et que souligne la chanson "Enfants qui ne connaissent pas la guerre ", placée en exergue de cette communication - grand succès des années 1970, bien qu'elle soit considérée comme pacifique, cette chanson révèle aussi un certain état de choses (Igarashi, 2000, 197). La génération née après la guerre qui ne l'a pas connue représente la possibilité de construction d'un pays forcément pacifique à la condition d'effacer les traces de la guerre, de la mémoire du passé récent. Ainsi cette chanson partage le discours idéologique où l'amnésie et l'oubli de la guerre sont nécessaires à la reconstruction du pays. Pendant les années 1960, le pays connaît un accroissement économique vertigineux qui commence dès les années 1950, en modifiant de façon significative l'espace urbain et la façon d'y vivre.

7 Les Jeux olympiques de Tokyo en 1964, en même temps qu'ils exhibent un «nouveau Japon » reflètent les signes de la guerre évités par le discours officiel. En se dissimulant, les signes se font présents en tant qu'absence. Avant la guerre, il était prévu que la ville accueillerait les jeux en 1940, mais ceci n'aura pas lieu à cause de la deuxième guerre 
mondiale. Dans ce contexte, le Japon était en guerre contre la Chine - qui a été violemment envahie et occupée, ainsi que d'autres pays asiatiques, même si ces épisodes ont été refoulés jusqu'à aujourd'hui et objets de conflits de reconnaissances et de réparations.

Dans Mystère Koumiko (1965), la première œuvre consacrée au Japon, tournée pendant les Jeux olympiques de Tokyo en 1964, l'idée de dépaysement est déjà présente. Les jeux sont, à leur tour, un événement important, signalant l'entrée du Japon dans la modernité. La ville de Tokyo et la notion d'inconnu apparaissent dans la figure d'une femme et à travers sa médiation. L'Autre mystérieux et inconnu s'incarne donc dans le visage d'une femme ; comme dans d'autres œuvres de Marker, la femme est la figure de l'altérité et l'image la plus cinématographique; «le cinéma et la femme sont restés comme deux notions inséparables, un film sans femme est comme un opéra sans musique » (Chris Marker: 1997). Dans Mystère Koumiko, l'énonciateur essaie de connaître cette femme, en lui posant des questions, alors qu'elle déambule dans les rues de Tokyo. La ville tokyoïte est le signe d'une transformation, du Japon de l'après-guerre à un pays modernisé - il s'agit d'un paysage renouvelé et d'un style de vie de plus en plus occidentalisé. Pour la première fois les Jeux olympiques avaient lieu hors de l'Occident, c'était une célébration officielle de l'entrée du Japon reconstruit dans la vie moderne, mais aussi un événement politique qui marque l'hégémonie américaine dans le contexte de la guerre froide, afin de garantir et "promouvoir la compréhension mutuelle des pays de l'Asie non-communiste" (Noriko Aso, 2002 :13). Un Japon futuriste apparaît aussi dans ces JO, le premier à utiliser des technologies avancées pour mesurer le temps, ainsi que des ordinateurs pour les statistiques. Tokyo a été soumis à des modifications radicales du projet urbain notamment par l'implantation de la première ligne de transport ferroviaire à grande vitesse - le Shinkansen - qui a été inaugurée à l'occasion des JO. Au début du film, une voix de reportage annonce, sur un plan où apparaît l'image de l'ouverture des Jeux à la Une d'un journal japonais écrit en anglais :

[...] Les dix-huitièmes Jeux olympiques de Tokyo ont commencé par une révérence, celle de l'Empereur Hirohito qui s'est incliné devant les emblèmes déployés. Le Japon attendait les Jeux olympiques depuis 1940 - mais ce n'est pas le même Japon. En 1940, les Japonais ne fabriquaient pas de transistors, et les Empereurs ne faisaient pas de révérence (Marker, $1967: 10)$

Les images suivantes sont un plan de visage d'une dame dans la rue et puis des femmes qui font le ménage. Même si le commentaire annonce le changement profond sur lequel cet événement officiel arrive, les images nous montrent le quotidien, la normalité. Enfin, après l'image de la flamme olympique, on retrouve la protagoniste Koumiko dans le stade. Les Jeux olympiques n'apparaissent qu'en arrière-plan du film, celui-ci étant centré sur Koumiko et sa flânerie autour de la ville. Toutefois, ce déplacement n'est pas uniforme, mais interrompu de temps en temps, donc l'espace n'est pas une extension successive, mais formé des signes entrecroisés dans une temporalité non-linéaire. Ainsi le passé de la guerre apparaît comme un fantôme qui hante le film dans son absence, même si les signes de la destruction comme l'occupation japonaise en chine et la seconde guerre mondiale ne sont pas présents directement. L'horreur du passé récent que l'histoire officielle et le mouvement de modernisation veulent effacer semble être un élément latent. Quand la caméra déambule en suivant Koumiko, le dialogue entre le cinéaste et la protagoniste porte sur l'identité japonaise, est-ce que Koumiko est complètement japonaise et quelle serait la signification de l'esprit japonais? Le 
montage nous montre les visages de gens dans la rue et les panneaux lumineux électroniques où on peut envisager l'image d'une chouette (animal du bestiaire markérien) et des lettres qui forment le nom « Kurosawa » (presque vingt ans plus tard Marker réalise le film A.K. en 1985 sur le cinéaste japonais pendant le tournage de Ran).

Les mêmes signes, les lumières électroniques des panneaux, les visages d'anonymes, la chouette de Ginza, évidemment les chats, le rythme de déambulation dans la ville de Tokyo présents déjà dans le premier film, se trouvent aussi dans Sans Soleil (1982) et aussi dans quelques images du livre de photographies intitulé justement Le dépays (1982). Dans ces deux œuvres, Koumiko est absente, mais les échos et les réverbérations de Mystère Koumiko habitent les œuvres. Comme les nombreuses séquences où Koumiko est dans le train, où on voit son visage qui a l'air de réfléchir sur des choses qui ne sont pas révélées, Le dépays est peuplé d'une séquence de photos de gens endormis dans les trains :

On peut passer des journées entières à naviguer de train en métro [...], avec des grands passages à vide qui permettent de choisir stratégiquement son angle et son vis-à-vis. Commence alors la chasse aux dormeurs. Ils te fascinent. Tu prends le train pour les voir, tu oublies tes rendez-vous,... pour rester quelques minutes de plus devant le court-métrage absolu, le gros plan idéal d'un visage de dormeur ou dormeuse. Leur sommeil libère une gamme d'expressions que la tenue sociale et le souci de l'apparence refrènent à l'état de veille, et tu peux lire sur ces visages endormis toute leur histoire, sourire et crispation, dodelinement et extase. (Marker, 1982)

11 À travers la photo, il cherche ces visages de dormeurs dans les trains, en poursuivant les signes où l'histoire individuelle se croise avec la collectivité, et rencontre celle qu'il appelle « la Derelitta " (l'abandonnée), après un tableau mystérieux de Boticelli qui a été rarement exposé au public, qui montre une femme qui pleure, dont on ne peut pas voir le visage. Le texte nous dit qu'il a suivi cette femme " pendant une heure,... toutes les saisons, les sautes rapides et confuses » (Marker, 1982), et qu'il a fait attention à son visage comme à celle d'un être aimé. Néanmoins, il avertit le lecteur à ne pas la chercher car ces photos n'ont pas été publiées, puisque faire cela "était la trahir » (Ibid.). Donc, encore une fois l'absence se fait présente, et exige du voyeur/lecteur qu'il l'imagine. Comme une mémoire archéologique, sur ce visage de la Derelitta, on peut trouver celui de Koumiko, puis ceux d'autres femmes «qui font battre le cœur ». Dans Immemory (1997), notamment Simone Genevois dans le film La merveilleuse vie de Jeanne d'Arc (1928), en tant que souvenir d'enfance est le signe d'un premier amour "quelque chose qui revenait sans cesse, qui se mêlait à tous les instants de la vie » (Marker, 1997) - qui était un gros plan au cinéma. Le texte d'Immemory nous dit aussi que le fait que ce film et ce visage aient été oubliés reste aussi comme un mystère inexplicable dans l'histoire du cinéma. Ainsi, voyager dans le train est aussi voyager dans le temps.

Dans Sans Soleil (1982), les trains sont toujours là, comme trajet qui unit espace, mémoire et imagination, car " Tokyo est une ville parcourue de trains, cousue de fils électriques, elle montre ses veines » (Marker, 1993 : 81). Pour Krasna, le cinégraphistepersonnage qui n'apparaît jamais et qu'on ne connaît que par les lettres filmées de ses voyages entre le Japon et l'Afrique envoyées à sa lectrice, la ville de Tokyo lui apparaît comme un rêve. Les galeries et les magasins forment une ville souterraine où tous les chemins conduisent aux lignes des trains, une ville doublée de rêves et d'images. Une séquence de ces galeries souterraines, les gens qui passent sans arrêt, des affiches 
publicitaires - parmi elles, une vitrine où il y a un mannequin vêtu en kimono placé entre deux grands masques du théâtre Nô - alternées entre elles, forment une imagerie de rêve surréaliste, comme si cette ville doublée était celle d'un songe. Ainsi le commentaire nous dit: «Je commence à me demander si ces rêves sont bien à moi, ou s'ils font partie d'un ensemble, d'un gigantesque rêve collectif dont la ville serait la projection" (Ibid. : 87). En fait, c'est comme s'il y avait une inversion, où la ville de Tokyo devenait une création, un rêve de la collectivité qui habite et passe par elle. Cette conception est proche de celle de Walter Benjamin, selon lequel l'investigation matérialiste de l'histoire de $\mathrm{XIX}^{\mathrm{e}}$ siècle développée dans Le Livre des Passages se faisait à travers l'étude des passages parisiens comme le passage de l'Opéra, le passage Vivienne, entre autres - galeries à l'abri de la rue pour le commerce - comme à Tokyo. Selon Benjamin :

La majorité des passages sont construits à Paris dans les quinze années qui suivent 1822. [...] Les magasins de nouveautés, premiers établissements qui ont constamment dans la maison les dépôts de marchandises considérables, font leur apparition. Ce sont les précurseurs des grands magasins. [...] Les passages sont les noyaux pour le commerce des marchandises de luxe. (Benjamin, $2006: 39-40$ ).

Il s'agit d'un nouvel endroit dans la ville, mais qui en tant qu'espace pourrait être aussi une représentation d'une époque - le XIX ${ }^{e}$ siècle - et d'un imaginaire, précurseur aussi, on peut le dire, des galeries tokyoïte du $\mathrm{xx}^{\mathrm{e}}$. Le passage, chez Benjamin, est un lieu représentatif de la ville et de la modernité, où ses images collectives sont formées par une interpénétration entre le nouveau et l'ancien (Ibid. : 41), ce sont des images de désir et de rêve. Cet éveil apparent dans la vie urbaine est faux car il se révèle comme un sommeil collectif, ainsi la ville, son architecture et ses passages sont l'intérieur de la collectivité avec ses rêves et délires inconscients, qui ne permettent pas à la conscience historique d'avoir lieu. Dans ce monde où tout apparait comme nouveautés, celles-ci ne sont que répétitions éternelles, le progrès technique n'ayant pas vraiment apporté d'amélioration à la vie humaine. En effet, l'humanité continue de vivre dans un immense songe illusoire où la domination se fait présente mais cache ses moyens de production. Selon Jean-Louis Déotte «le passage parisien aura été le lieu où le $\mathrm{XIX}^{\mathrm{e}}$ siècle aura rêvé et enfanté le $\mathrm{xx}^{\mathrm{e}}$ siècle " (Déotte, 2008, p. 10), dans lequel toutes les réalités s'étaient transformées en fantasmagories, où « si le psychosocial perdure, c'est sur le mode de l'onirique »(Ibid.). Il fallait donc arrêter cette temporalité onirique, d'une évolution apparente qui n'est pas vraie, en se réveillant de ces rêveries. Chez Benjamin il y a un fort rapport entre l'acte de se réveiller et la remémoration ; le passé, le présent et le futur sont liés par le réveil.

Dans Sans Soleil, Marker présente aussi l'idée d'un grand rêve collectif, aux images de galeries souterraines, se succèdent des images de gens qui donnent leurs billets de train pour embarquer, et l'on constate la répétition des gestes, ainsi que la gestualité de ceux qui achètent leur billet dans la machine ; le commentaire de ces images nous dit : « Le train peuplé de dormeurs assemble tous les fragments de rêve, en fait un seul film, le film absolu. Les tickets automatiques deviennent des billets d'entrée " (Marker, 1993 : 87). Donc, il nous montre une association entre les billets de train et les billets à l'entrée d'une salle de cinéma, où on va voir ce film absolu d'un rêve collectif qu'est la ville. D'ailleurs, la séquence acquiert une consistance onirique, puisque le son d'un train qui entre en marche est accompagné d'une image d'un chemin de fer issu d'un dessin animé "mangá » japonais, alterné avec des plans tournés dans la ville de différents chemins de fer et de stations toujours selon le même cadrage d'un travelling 
avant, comme si l'on était dans le train en voyant ce chemin imaginaire composé de plusieurs parties d'images photographiques et dessinées. En plus de cette séquence d'un chemin de fer imaginaire, il y a une composition similaire dans Mystère Koumiko, à la fin du film, où des chemins de fer différents forment un trajet lorsque la protagoniste répond à la dernière question posée à propos de l'histoire, de la guerre et comment cela pourrait influencer sa vie. De façon que ce chemin construit de plusieurs trajets se présente comme une route temporelle, vers l'histoire, où la mémoire individuelle se rattache à la collectivité, selon la parole de Koumiko :

Toujours, tous les jours, tous les soirs, tous les matins, toujours, quelques choses ont lieu, n'importe quelles choses. Elles arriveront une par une, sur la ligne de l'histoire humaine. Mais pour moi, ce sont des incidents de chaque matin, qui sont jetés par la porte. [...]. Juste à la même époque, l'humain était sur le point de souffrir ; ils sont allés à la guerre, [...] ils ont crié... et les chairs humaines sont déchirées. [...] Mais bientôt ils arriveront, les résultats des événements. C'est comme la vague de la mer, une fois qu'il arrive un tremblement de terre, même si c'est un accident lointain, la vague avance peu à peu et cela finit par arriver jusqu'à moi. (Marker, 1967 :36)

Par ailleurs, c'est comme si Koumiko était endormie, sans rien savoir de l'histoire et de la guerre, ainsi que le peuple japonais qui voulait effacer cet événement de la mémoire. Si le sujet de la guerre apparaît de façon très allusive dans ce premier film, il est plus présent dans Sans Soleil et devient le thème principal de Level Five (1997). Comme si dans ces réverbérations d'un film à l'autre, un tremblement de plus en plus fort se faisait ressentir jusqu'à aboutir au sujet de la bataille d'Okinawa. Cet événement peu connu de la seconde guerre mondiale provoqua la mort de nombreux civils sur cette île, notamment lors d'un grand suicide collectif causé par des annonces officielles des autorités japonaises. Un grand sommeil en tant qu'oubli collectif s'impose aux japonais, depuis Mystère Koumiko. Dans Level Five, Marker apparaît comme un des énonciateurs et il parle d'une amnésie collective japonaise qui s'abat aussi sur lui-même dès qu'il devint trop proche du Japon, presque un Japonais. Ainsi, on observe une dialectique de la distance et de la proximité par rapport au temps et à l'espace, car celui qui est trop proche ne peut pas apercevoir, il devient dormeur qui donne continuité à la répétition, alors que la distance permet le réveil et la prise de conscience. Pour Koumiko, il fallait du temps pour comprendre l'existence de la guerre et ses horreurs, ainsi que le fait que chaque événement historique, même lointain dans le temps ou l'espace apporte leurs conséquences. Dans Level Five, l'absence fantasmatique de la guerre qui hante les films précédents devient le centre de réflexion, justement à partir d'Okinawa, cette île lointaine, oubliée par le japonais et qui n'est pas considérée comme partie du "vrai » Japon, c'est l'altérité sacrifiée dans le pays lui-même.

Enfin dans Sans Soleil, après le trajet de train onirique, nous assistons à une séquence de plusieurs dormeurs et dormeuses, des gens qui sont en train de sommeiller, de succomber peu à peu au sommeil. Puis, les images de dessins animés reviennent et le train part vers le ciel. Après un plan sur le visage d'une dormeuse, une série d'images de la télévision japonaise avec ses fantômes et monstres - une poupée dont la tête plane au-dessus du corps, les yeux effrayés, les films de samouraï- sont montrées en alternance avec les images des dormeurs. De sorte que ce grand rêve collectif et fragmentaire est fait d'images médiatiques de la télévision avec les créatures imaginaires fantomatiques des films de terreur japonais. À la fin, dans une scène qui nous rappelle le réveil de la femme dans La Jetée (1962), on voit une femme endormie qui ouvre les yeux, mais dans Sans Soleil, il n'y a pas de changements, pas d'interruptions d'un régime d'images. Ensuite se présente l'image d'un train qui passe, 
et derrière celui-ci, une affiche publicitaire avec de grands yeux dessinés qui semblent nous regarder, comme si ces images rêvées nous surveillaient aussi, mais d'une façon inégale, car les images - de la télévision, des films, de la publicité - deviennent plus réelles que la réalité. Le sommeil médiatique de la société du spectacle (Guy Debord, 1997), substitue la vie par l'imagerie et la rêverie de l'industrie culturelle. De sorte que l'image ne se révèle pas comme représentation, mais prend la place de la réalité. Le réveil dans cette partie du film nous rappelle la continuité du domaine des images et des simulacres partout où il n'y a pas de distance entre les spectateurs et les images, de telle façon qu'on ne s'aperçoit plus de la réalité en la confondant avec les images. Toutefois, de cette façon, le film annonce cet oubli, cette tromperie de l'image, surtout quand il nous montre les images transformées, solarisées et modifiées par le synthétiseur de Hayao Yamaneko :

[...] si les images du présent ne changent pas, changer les images du passé [...] Des images moins menteuses... que celles que tu vois à la télévision. Au moins elles se donnent pour ce qu'elles sont, des images, pas la forme transportable et compacte d'une réalité déjà inaccessible (Marker, 1993 : 86).

17 Pour conclure, à travers cette mise en scène, Sans Soleil présente une réflexion sur sa propre matérialité en tant qu'image, pour se réveiller de son propre songe de cet univers trompeur de l'image. En même temps, il s'agit d'une réflexion sur la mémoire et sur sa représentation comme image dans une histoire contemporaine de plus en plus médiatique. Ainsi, chez Marker, le Japon est ce « dépays » qui en nous dépaysant, nous réveille dans le sommeil par des images cinématographiques pour les apercevoir comme telles, où le temps et l'espace se croisent.

\section{BIBLIOGRAPHIE}

Aso Noriko (2002), "Sumptuous Re-Past: The 1964 Tokyo Olympics Arts Festival”, in Positions: East Asia Cultures Critique, 10, $\mathrm{n}^{\circ}$ 1, Spring 2002, p. 7-38.

Benjamin Walter (1993), “Sobre o conceito da história", in Obras Escolhidas vol. 1 - Magia e técnica, arte e política ( 5 éd.), São Paulo, Brasiliense, p. 222-232.

Benjamin Walter (1997), "Infância em Berlim por volta de 1900", in Obras Escolhidas vol. II - Rua de mão única, J. C. M. Barbosa, (trad.), São Paulo, Brasiliense, p. 71-142

Benjamin Walter (2006), Passagens, Belo Horizonte/ São Paulo, Ed. UFMG/Imprensa Oficial.

Bolle Wille (2000), Fisiognomia da metrópole moderna, São Paulo, Edusp.

Buck-Morss Susan (2002), Dialética do olhar - Walter Benjamin e o projeto das Passagens, Belo Horizonte/ Chapecó, SC, Ed. UFMG/ Ed. Universitária Argos.

Cardoso Sérgio (1989), “O olhar dos viajantes”, in Novaes, Adauto (org.), O Olhar, São Paulo, Companhia das Letras, p. 347-360.

Debord Guy (1997), A sociedade do espetáculo, Rio de Janeiro, Contraponto. 
Déotte Jean-Louis (2008), « La ville appareillée : Arendt, Benjamin et Baudelaire », Revue Appareil [En ligne], numéro spécial 2008, http://revues.mshparisnord.org/appareil/index.php ?id =449

Dubois Philippe (2002) (ed.), Theoreme 6 - Recherches sur Chris Marker, Paris, Sorbonne Nouvelle. Guy Gauthier (2001), Chris Marker, écrivain multimédia, ou voyage à travers les médias, Paris, L 'Harmattan.

Grélier Robert (1986), O Bestiário de Chris Marker, V. Bobichon (trad.), Lisboa, Livros do Horizonte. Igarashi Yoshikuni (2000), Bodies of memory - narratives of war in postwar Japanese culture, 1945-1970, New Jersey, Princenton University Press.

Matos Olgária (1997), “Espaço e Tempo : a cidade e a história viajante”, in História viajante notações filosóficas, São Paulo, Studio Nobel, p. 139-150.

Marker Chris (1967), « Le Mystère Koumiko », in Commentaires II, Paris, Seuil, p. 8-37.

Marker Chris (1982), Le dépay, Paris, Herscher.

Marker Chris (1993), « Sans Soleil », in Trafic, $\mathrm{n}^{\circ}$ 6, printemps 1993, p. 79-97.

Marker Chris (1997), Immemory cd-rom, Paris, Centre Pompidou.

Möller Olaf (2003), "Ghost world - Japan through the looking glass", in Film Comment, July/ August 2003, p. 35-37.

Selimann-Silva Marcio (2003), “Catástrofe, História e Memória em Walter Benjamin e Chris Marker: a escritura da memória", in História, Memória e Literatura - O testemunho na Era das Catástrofes, Campinas, SP, Éd. UNICAMP, p. 391-417.

\section{RÉSUMÉS}

Cet article propose de réfléchir sur le statut du Japon, en tant que territoire où se croisent la mémoire, l'imaginaire, le temps et l'espace - lieu de l'Altérité et du dépaysement - dans les œuvres de Marker, notamment: Le mystère Koumiko (1965), Sans Soleil (1982), Le Dépays (1982), Level Five (1997). Il s'agit d'analyser comment ces œuvres nous donnent une vision du Japon de l'après-guerre, affligé d'une amnésie collective et par la présence des images médiatiques.

This article focuses on the status of Japan, as a place where memory, imagination, time and space are related - a space of alterity and displacement - in Marker's works, specifically: Le mystère Koumiko (1965), Sans Soleil (1982), Le Dépays (1982), Level Five (1997). This treatement aims to analyse how these works depicted post-war Japan - afflicted by collective amnesia and the by presence of media images.

\section{INDEX}

Personnes citées : Marker (Chris), Benjamin (Walter)

Mots-clés : Japon 
AUTEUR

EMI KOIDE

Doctorante à l'Institut de psychologie de l'université de São Paulo (Brésil) en cotutelle avec l'université de Paris 8 (axe de recherche : Appareil, esthétiques, arts et industries), boursière FAPESP (Fondation d'Appui à la Recherche de l'État de São Paulo), koide.emi@gmail.com 